\title{
Congenital factor XII deficiency
}

INSERM

\section{Source}

INSERM. (1999). Orphanet: an online rare disease and orphan drug data base. Congenital factor XII deficiency. ORPHA:330

Congenital factor XII deficiency is an autosomal recessive systemic dysfunction of the hemostatic pathway, that is due to a defect in the coagulation factor XII (FXII or Hageman factor), and is either asymptomatic or characterized by a prolonged activated partial thromboplastin time and an increased risk for thromboembolism. FXII deficiency is strongly associated with primary recurrent abortions. 